\title{
The effect of structural-kinetic features of hologram formation on holographic properties of photopolymers
}

\author{
T. Smirnova, O. Sakhno*, S. Lozenko \\ Institute of Physics NAS of Ukraine, 46, prospect Nauky, 03028 Kiev, Ukraine \\ Phone: +380 (44) 26512 20, fax: +380 (44) 2651752 \\ *Fraunhofer Institute AG Polymerphotochemie Geiselbergstr. 69, D-14476 Golm \\ Phone: 0331568 1259, fax: 0331568 3259, E-mail smirnova@iop.kiev.ua
}

\begin{abstract}
A thermodynamic model of the holographic recording process in photopolymers have been developed. By the example of photopolymerizing compositions PPC-488 containing oligoetheracrylates and neutral components (NC) we have explored dependencies of holographic characteristics of medium on NC concentration and thermodynamic properties of polymer-NC system. The thermodynamic affinity of the polymer forming during recording and NC was evaluated using the difference of their solubility parameters $\Delta \delta_{P, N C}$. We have determined the feasible range of variation for $\Delta \delta_{P, N C}$ and optimal concentration of $\mathrm{NC}\left(N_{0}^{\text {opt }}\right)$ that ensure high-performance recording. It was ascertained that excess in NC concentration over the optimal value leads to the increase of photoinduced light scattering in the layer. The efficiency of scattering depends on the size of microphase enriched by NC. We have determined the size of microphase particles and its dependence on kinetic parameters of polymerization.
\end{abstract}

Keywords: photopolymer holographic materials, holographic recording, holographic grating, thermodynamic model.

Paper received 23.04.04; accepted for publication 21.10.04.

\section{Introduction}

Photopolymer systems possess many advantages as attractive recording materials for $3 \mathrm{D}$ holography. Originally two approaches to the creation of photopolymer recording materials were proposed: 1) a holographic film that is a neutral polymer matrix containing polymerizable compounds [1], or 2) a liquid mixture of components that is cured during the recording [2]. Holograms recorded in solid films, as a rule, require optical or thermal development and fixing. Liquid compositions are so-called selfdeveloping media. They ensure the formation of holograms during recording and are characterized by the simplest production process of the medium and recording layer. Nowadays liquid compositions are widely used for creation of polymer-liquid crystal $[3,4]$, and polymernanoparticle $[5,6]$ periodical structures.

Liquid photopolymerizable compositions (PPC) include two monomers that differ in reactivity and refractive indices and initiator of radical photopolymerization. Stable holograms in photopolymers are formed as a re- sult of the diffusive redistribution of components in the course of inhomogeneous polymerization in the interference pattern. In liquid compositions both monomers must take part in diffusion process to create stable hologram. In the limiting case the monomer with lower reactivity can be substituted by a neutral component (NC), which does not take part in the chemical reaction but is involved in the diffusion.

The present article studies liquid PPC developed in the Institute of Physics NAS Ukraine [7-9] containing oligomers and neutral components. We have shown that phase separation of the initial mixture plays significant role in hologram formation [10-12]. We have also proposed a thermodynamic model of recording process. The purpose of the present work is the development of thermodynamic approach to holographic recording in PPC. We analyze dependence of holographic characteristics of PPC on thermodynamic properties of system and consider influence of microstructure of medium on its holographic properties. It should be noted that in media containing two monomers, the low-reactive monomer polym- 


\section{T. Smirnova et al.: The effect of structural-kinetic features of hologram formation on ...}

erizes when diffusive separation of components is finished. Therefore, displacement of this monomer with NC does not influence on generality of results.

\section{Formation of the phase structure}

The PPC-488 and its modifications are binary compositions containing oligomers, which form a three-dimensional polymer network. Since the polymer network swells to some extent in its own monomer and different solvents, polymer formation gives rise to the displacement of solvent from the network. Therefore polymerization of monomer-NC mixture under uniform illumination results in formation of two-phase structure: the dispersion of microdrops of $\mathrm{NC}$ with polymer molecules dissolved ( $\beta$-phase) in a polymer matrix with equilibrium content of NC ( $\alpha$-phase). Polymerization in an interference pattern has additional features (Fig. 1). Initially, monomer polymerizes in the regions of maximum illumination. The polymer being formed has limited thermodynamic compatibility with the NC. For this reason NC is forced out of high intensity regions and forms microdrop structure mainly in low intensity zones. Fig. 1 shows a two-phase structure obtained when polymer does not swell in the NC and the latter is completely displaced out of the polymer network. Relatively low concentration of NC in the $\alpha$-phase and, accordingly, a low concentration of polymer molecules in the $\beta$-phase enriched by NC are typical for a three-dimensional polymer network. A microscopic picture of grating with period $\Lambda=50 \mu \mathrm{m}$ confirms the two-phase structure of holograms in PPC (Fig. 2). When polymerization is relatively slow and inter-diffusion between the nuclei of new phases has time to restore the equilibrium, the "pathway" of phase separation in coordinates $\gamma_{P}$ versus $\varphi_{N C}$ (depth of polymer conversion versus normalized concentration of NC) can be described by a binodal curve [13]. If the properties of a polymer network depend on intensity, phase diagrams for the mixture in illuminated and dark zones will be different.

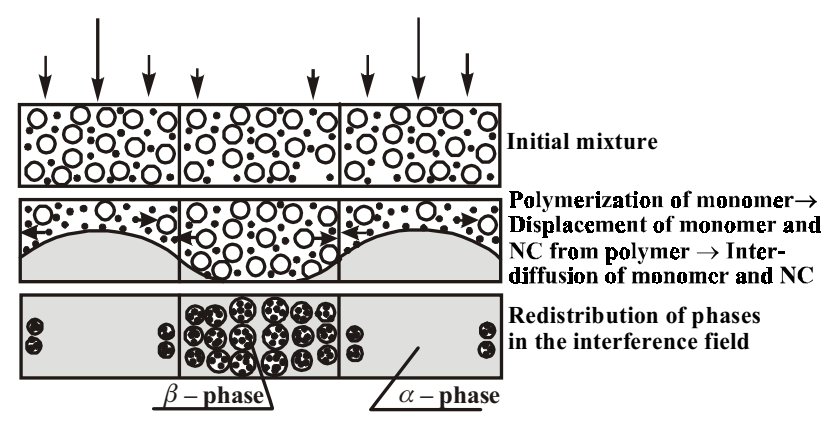

Fig. 1. Principal scheme of two-phase structure formation in the course of polymerization within the interference pattern.

$\mathrm{O}$, - molecules of monomer and $\mathrm{NC} ; \square-$ polymer network.

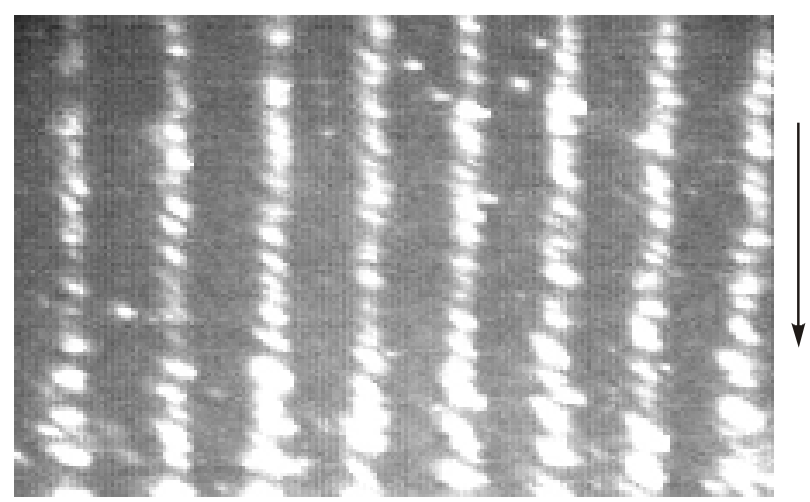

Fig. 2. Microscopic image of the grating with $\Lambda=50 \mu \mathrm{m}$. (An arrow indicates the decrease of field intensity)

Within this framework the hologram in a binary medium may be treated as a spatially organized heterogeneous structure with periodic distribution of phases. Such two-phase system is thermodynamically at quasi-equilibrium and diffusion of components between phases is forbidden, thus establishing a permanently stable hologram.

\section{Dependence of holographic characteristics on thermodynamic properties of mixture}

As it was shown in $[11,12]$, the amplitude of the refractive index modulation in a holographic grating that is a twophase structure can be described by the equation:

$$
\begin{aligned}
& n_{1}=\frac{1}{2}\left|\left(n_{\alpha}-n_{\beta}\right) \cdot\left(v_{\beta}^{\max }-v_{\beta}^{\min }\right)\right| \rightarrow \\
& \rightarrow \frac{1}{2}\left|\left(n_{P}-n_{N C}\right) \cdot\left(v_{\beta}^{\max }-v_{\beta}^{\min }\right)\right|
\end{aligned}
$$

where $n_{\alpha}, n_{\beta}, n_{P}, n_{N C}$, are the refractive indices of corresponding phases, polymer and $\mathrm{NC} ; v_{\beta}^{\max }, v_{\beta}^{\min }$ are volume fractions of the $\beta$-phase in the light and dark zones, respectively.

The value of $n_{1}$ increases with increase of segregation of the polymer and the NC. The degree of segregation is determined by thermodynamic affinity of components, kinetic parameters of polymerization reaction and by their diffusive separation. The value of $\left|\left(n_{\alpha}-n_{\beta}\right)\right|$ reaches its maximum, if the interdiffusion rate allows transfer of components over a distance that is equal to the average distance between nuclei of $\alpha$ and $\beta$ phases. When segregation of components is large, $\left|\left(n_{\alpha}-n_{\beta}\right)\right|$ approaches the difference between refractive indices of the polymer and the $\mathrm{NC}\left(\Delta n_{P, N C}\right)$. The second term in equation (1) is determined by concentration of $\mathrm{NC}\left(N_{0}\right)$ in the mixture and also by the interdiffusion rate. It reaches the maximum value, if the system allows the mass-transfer over a distance of $\Lambda / 2$. Thus, when diffusion does not limit the material redistribution, holographic properties of PPC are determined by thermodynamic compatibility of the polymer - NC system. 
Research of the recording kinetics of gratings with $\Lambda \leq 2 \mu \mathrm{m}[14]$ has shown that while using the low-molecular NC diffusive redistribution of components finishes before the layer is fully polymerized. In this case, the diffusion process does not limit the recording rate that is determined by the rate of polymerization.

The thermodynamic affinity of polymer and NC can be determined by the difference between their solubility parameters $\left(\Delta \delta=\mid \delta_{P}-\delta_{N C}\right)[15,16]$. The solubility parameter, $\delta=\left(\Delta E_{i} / v_{i}\right)^{1 / 2}$, where $\Delta E_{i} / v_{i}$ is the density of cohesion energy (the evaporation energy per mole). The equality $\delta_{S}=\delta_{P}$ implies that the $S$ - and $P$-components are mutually soluble. Because cross-linked polymers are not soluble, this condition corresponds to the maximum possible swelling of polymer network in a solvent. Since this equality implies an athermic dilution, it is generally assumed that the solvent is suitable, if $\left|\delta_{S}-\delta_{P}\right|<$ $<2.5-3 \mathrm{MPa}^{1 / 2}$. Ability to form hydrogen bonds widens this region. The solubility parameter, $\delta$, is available in literature for the large number of polymers and organic liquids. It also can be calculated or measured by simple methods. It is evident from this model that for effective recording NC should not be a "good solvent" for the polymer.

The dependence of the holographic characteristics on the thermodynamic properties has been determined by measuring the dependence of $n_{1}$ on $N_{0}$ for NC with different $\delta_{N C}$ (Table 1). For this purpose, transmission gratings with $\Lambda=0.8 \mu \mathrm{m}$ and $d=25 \mu \mathrm{m}$ were recorded. Recording was carried out using $\operatorname{Ar}$ laser $\left(\lambda_{r}=0.488 \mathrm{~mm}\right)$ with $I=$ $=1 \mathrm{~mW} / \mathrm{cm}^{2}$. Note that for the sample with this thickness the dynamic range $n_{1}^{\max } \cong 0.01$ ensures the diffraction efficiency of $\eta \cong 1$.

The following regularities were obtained. Stable gratings are formed, if the concentration of NC in initial mixture, $N_{0}$, exceeds the NC equilibrium content of polymer $N_{0}^{0}$. An optimum NC concentration, $N_{0}^{o p t}$, exists that

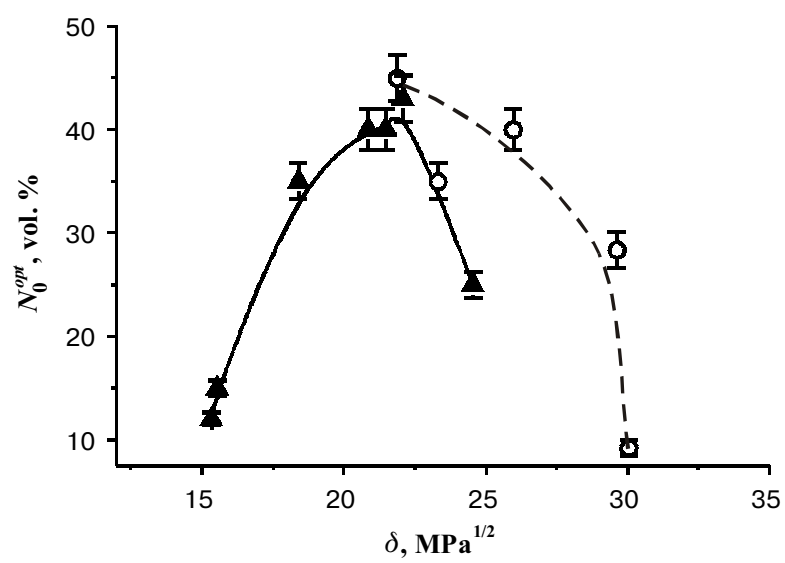

Fig. 3. Dependence of NC optimal concentration on its solubility parameter.

ensures the maximum dynamic range of recording. The decrease of $n_{1}$ with increase of $N_{0}$ above the optimum value is the result of increased light scattering in hologram due to the growth of the size of $\beta$-phase particles (see sec. 4). In Fig. 3, the change of $N_{0}^{o p t}$ is plotted versus $\delta_{N C}$ for NC, listed in Table 1 . The neutral components No.8-12 can create hydrogen bonds. The value of $\delta_{P}$ is about $20.5 \mathrm{MPa}^{1 / 2}$. Since $N_{0}^{\text {opt }}$ depends on the size of microstructure, the curves in Fig. 3 depict only a general trend of $N_{0}^{o p t}(\delta)$ change. The optimum concentration decreases with a decrease in thermodynamic affinity between the polymer and $\mathrm{NC}\left(\Delta \delta_{P, N C}\right.$ increases). This is obvious from the equation (1). The enhancement of component segregation due to the decrease of their compatibility causes the growth of the difference between the refractive indices of $\alpha$ and $\beta$ phases. As a result, the maximum value of $n_{1}$ can be achieved for smaller differences in the volume fraction of the $\beta$-phase in the "fringes" of the grating and, accordingly, at a smaller initial concen-

Table 1. NC characteristics and diffraction efficiency of gratings at the optimal concentration of NC.

\begin{tabular}{|c|l|c|c|c|c|c|}
\hline \hline No & NC & $n_{N C}$ & $n_{P}-n_{N C}$ & $\delta_{N C}, \mathrm{MPa}^{1 / 2}$ & $N_{0}^{\text {opt }}$, vol. $\%$ & $\eta$ \\
\hline 1 & Acetonitrile & 1.344 & 0.176 & 24.3 & 25 & 0.95 \\
\hline 2 & $\alpha$-Naphthalenebromide & 1.66 & -0.140 & 21.7 & 45 & 0.98 \\
\hline 3 & Pentachlorodiphenyl & 1.636 & -0.116 & 21.5 & 40 & 0.90 \\
\hline 4 & Quinoline & 1.627 & -0.107 & 21.3 & 40 & 0.85 \\
\hline 5 & Toluene & 1.467 & 0.023 & 18.2 & 35 & 0.42 \\
\hline 6 & $n$-Heptane & 1.387 & 0.133 & 15.5 & 15 & 0.95 \\
\hline 7 & $n$-Hexane & 1.375 & 0.145 & 14.9 & 12 & 0.95 \\
\hline 8 & Ethylene glycol & 1.432 & 0.089 & 29.9 & 7 & 0.35 \\
\hline 9 & Methanol & 1.328 & 0.193 & 29.7 & 25 & 0.80 \\
\hline 10 & Ethanol & 1.362 & 0.159 & 26 & 40 & $0.88^{1)}$ \\
\hline 11 & Butanol-1 & 1.399 & 0.122 & 23.3 & 35 & $0.95^{1)}$ \\
\hline 12 & Triethylene glycol & 1.456 & 0.065 & 21.9 & 45 & 0.80 \\
\hline
\end{tabular}

1) The strength of grating, $\pi n_{1} d / \lambda_{t} \cos \theta_{B}>\pi / 2$. 


\section{T. Smirnova et al.: The effect of structural-kinetic features of hologram formation on ...}

tration of NC. The value of $N_{0}^{o p t}$ is maximized when $\delta_{N C} \cong \delta_{P}$, because at this condition the equilibrium NC content in the polymer network is also a maximum.

Equation (1) can be used to estimate the minimum value of $N_{0}$ that provides a required dynamic range. If the neutral component is assumed to be completely displaced into dark regions,

$N_{0 \min }^{o p t} \sim n_{1} /\left|n_{P}-n_{N C}\right|$.

For $n_{1}^{\max } \geq 0.01$ and $\left|n_{P}-n_{N C}\right| \cong 0.1$ the minimum optimal concentration is about 0.1 .

Hence, for effective holographic recording $\left(n_{1}^{\max } \geq\right.$ $\geq 0.01)$ the values of $\Delta \delta_{P, N C}$ and $\Delta n_{P, N C}$ should satisfy the following conditions:

$\Delta n_{P, N C} \geq 0.1$

$\Delta \delta_{P, N C} \leq 5 \mathrm{MPa}^{1 / 2}$, if hydrogen bonds

are not formed,

$\Delta \delta_{P, N C} \leq 12 \mathrm{MPa}^{1 / 2}$ if hydrogen bonds can be formed.

Note that variations in $\delta_{N C}$ should not result in a violation of condition $\delta_{N C} \cong \delta_{M}$. Otherwise phase separation will take place in the initial mixture.

The rate of recording also depends on the thermodynamic affinity of the components. For materials of PPCtype, a reduction of this affinity allows to decrease the concentration of $\mathrm{NC}$ that increases both the polymerization and recording rates.

Finally it should be noted that these concepts are not only valid for materials of PPC-type. Phase separation occurs during holographic recording in compositions containing liquid crystals and nanoparticles.

\section{Influence of micro-heterogeneity of the photopolymer composition on its holographic properties}

It was noted above that the values $N_{0}^{o p t}$ and, correspondingly, $n_{1}$ are bounded by the size of $\beta$-phase particles. In this section experimental results are considered, that confirm this assumption. Light scattering was measured in PPC layers that contain different NC. Also, the size of $\beta$-phase particles was determined using microscopic measurements.

Integral variation of light scattering in a recording layer was estimated by the change of transmission for the probing He-Ne laser beam in the course of photopolymerization of PPC layer by incoherent spatially-homogeneous UV-radiation. The use of incoherent radiation excludes the recording of noise holograms [17]. Transmission of the layer was calculated as $T(t)=P_{t r}(t) / P_{0}(t)$, where $P_{t r}$ is the power of the passing beam, $P_{0}$ is the power of the falling beam.

Kinetics of transmission change was measured during polymerization for PPC with various NC concentrations. The results for two NC $\alpha$-bromnaphthalene which demonstrates high affinity to a polymer and $n$-hexane with low affinity are shown in Figs 4, 5. To exclude reflection and light scattering losses in substrate, the data

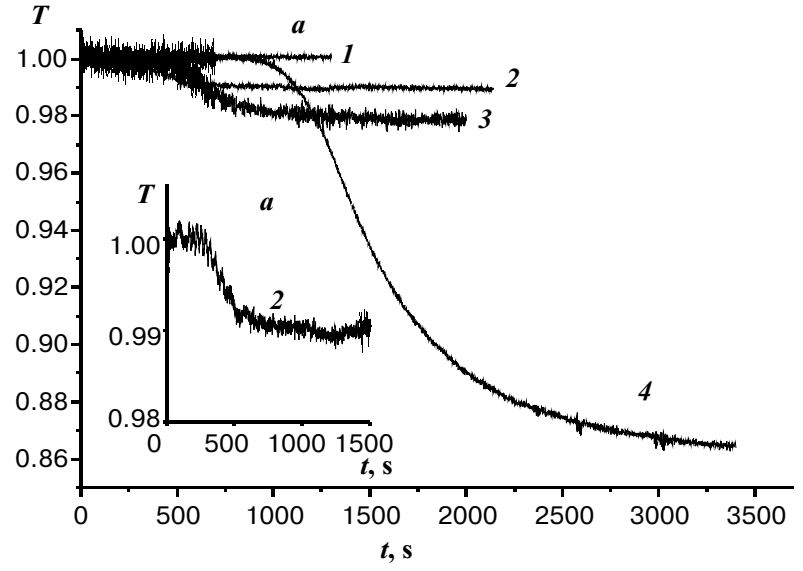

Fig. 4. The change of transmission of PPC layer with $\alpha$-bromnapthalene during polymerization by UV-radiation at concentration of $\mathrm{NC} 0$ (1), 40 (2), 60 (3) and 80 vol.\% (4).

was normalized so that the transmission of the initial liquid layer was $100 \%$. The error in kinetic curve measurement is less than $0.2 \%$.

In both cases, the transmission of the layer decreases with the increase of the NC concentration. However, when concentrations are equal, the terminal transmission substantially depends on the nature of NC. Thus, if for abromnaphthalene the increase of concentration up to $40 \%$ leads to decrease in transmission by $1 \%$ while injection of $n$-heptane decreases the layer transmission up to $35 \%$. The similar behavior was obtained for NC that form hydrogen bonds.

Examination of the structure of polymer layers with microscope Polam I-411 (1000x magnification) allowed determining the following behaviour. The structure of polymer composition contains discrete microinhomogeneities, which size increase with the increase of $\mathrm{NC}$ concentration. The size differs greatly for PPC with diffe-

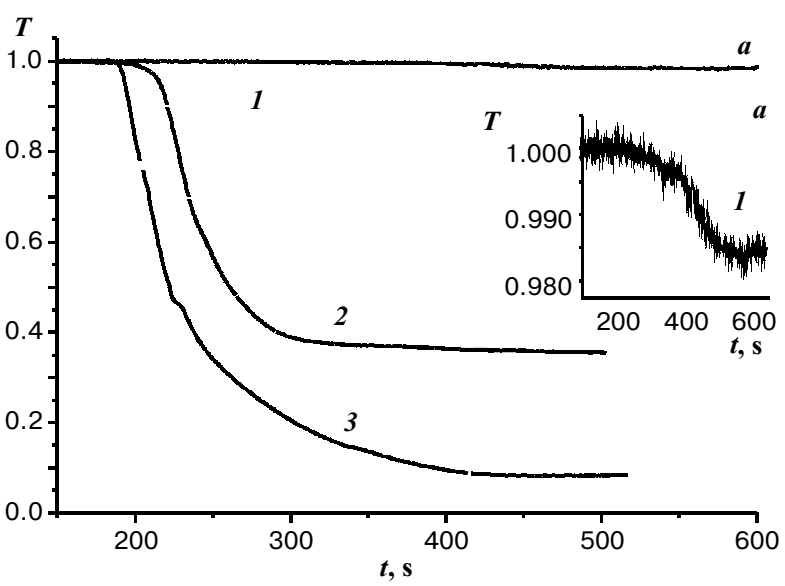

Fig. 5. The change of transmission of PPC layer with $n$-heptane during polymerization by UV-radiation at concentration of $\mathrm{NC}$ 12 (1), 20 (2) and 40 vol.\% (3). 
rent NC. When employing $\alpha$-bromnaphthalene the structure becomes distinguishable at $N_{0} \cong 60$ vol. $\%$. We can mark out the particles of two characteristic sizes: the separate micro-inhomogeneities with the diameter $D \approx 10 \mu \mathrm{m}$ and a substantial number of particles with $D<1 \mu \mathrm{m}$. When employing $n$-hexane as NC, the large-scale drops of emerging phase with characteristic diameters 5 and $100 \mu \mathrm{m}$ become visible at $N_{0}=20 \mathrm{vol} . \%$ They merge in some areas forming tree-type structures.

To determine the size of inhomogeneities in PPC that contains $\alpha$-bromnaphthalene, the turbidity spectrum method was used [10]. It was shown that at $N_{0} \cong 60 \mathrm{vol} . \%$ the radius of the scattering particle is nearly $0.75 \pm 0.2 \mu \mathrm{m}$. If the concentration of $\mathrm{NC}$ decreased to the optimal value of $45 \mathrm{vol} . \%$, precision of the method was insufficient to determine the size of the particles. These results coincide with the data of microscopic analysis and verify that prevailing size of the particles is $\leq 1 \mu \mathrm{m}$, when concentration of $\mathrm{NC}$ is about $60 \%$. The size of the scattering centers is much smaller than $1 \mu \mathrm{m}$, when concentration of $\alpha$-bromnaphthalene is optimal. This conclusion is confirmed by the fact of recording of high-effective reflective gratings with $N=6000 \mathrm{~mm}^{-1}$ [18]. Maximum diffraction efficiency $\eta \cong 1$ can be accomplished when the size of phase structure is less than $\Lambda / 2 \cong 0.08 \mu \mathrm{m}$.

So the investigation carried out reveals that the decrease of the recording efficiency with the increase of $N_{0}$ starts at the same values of $N_{0}$ as the increase of light scattering caused by enlargement of heterostructure particles. The increase of light scattering during recording leads to amplification of noise holograms that forms as a result of interference between recording and scattered waves. Amplification of noise hologram results in decrease of grating diffraction efficiency.

With the increase of compatibility of components, the decrease of $n_{1}$ is observed under a more excess of $N_{0}$ over the optimal value. This makes for an increase in the material dynamic range. In most of considered cases, the tendency to increase in $N_{0}^{o p t}$ with the increase of thermodynamic compatibility of components takes place. However, the values $N_{0}^{o p t}$ for different $\mathrm{NC}$ with the same solubility parameters can differ significantly. To illustrate this, let us compare these values for $\alpha$-bromnaphthalene and butanol-1. For $\alpha$-bromnaphthalene $\delta_{N C}=21.7 \mathrm{MPa}^{1 / 2}$, $N_{0}^{0} \approx 12$ vol. $\%, N_{0}^{o p t} \approx 45$ vol. $\%$; for butanol-1 $\delta_{N C}=$ $=23.3 \mathrm{MPa}^{1 / 2}, N_{0}^{0} \approx 16$ vol. $\%, N_{0}^{\text {opt }} \approx 30$ vol. $\%$. Increase in the equilibrium content of NC for $\alpha$-bromnaphthalene in comparison with butanol-1 can be explained by specific interaction of polymer and $\mathrm{NC}$, because butanol-1 can form hydrogen bonds. The decrease of the optimal concentration is a result of enlargement of $\beta$-phase particles. It is confirmed by measurements of light-scattering in a layer. Thus, transmission of $50 \mu \mathrm{m}$ thick polymer layer obtained as a result of polymerization of the composition with 40 vol. $\%$ of $\alpha$-bromnaphthalene exceeds $99 \%$, whereas for the layer with the same concentration of butanol-1 it is about $92 \%$. On the other hand, injection of ethanol with $\Delta \delta_{P, N C}$ higher than that of butanol-1 lowers $N_{0}^{0}$ down to 14 vol.\% and rises the optimal con- centration up to 40 vol. $\%$. Transition of the layer also increases up to $99 \%$.

It is known that the size of $\beta$-phase microdrops depends significantly on the polymerization rate. It was shown for oligoetheracylates that slowing of polymerization is accompanied by enlargement of phase particles and could be followed by full separation of a system [19]. We do not consider the influence of polymerization rate in details. However, while examining the microstructure of the gratings with $\Lambda=50 \mu \mathrm{m}$ recorded with gaussian beam, the increase of microdrops in size was observed at the periphery of gratings where the intensity of the recording field is lower (Fig. 2).

Thus, if the entire volume of $\beta$-phase displaced from the polymer is determined by thermodynamic compatibility of polymer and $\mathrm{NC}$ as well as the initial concentration of $\mathrm{NC}$, the characteristic size of $\beta$-phase particles depends on the total volume of displaced phase, polymerization rate and interphase tension forces.

Since microphase structure of polymer determines the resolution of PPC and noise characteristics of holograms, the possibility to control the size of microphase particles is quite important.

\section{Conclusions}

Thermodynamic approach to holographic recording in PPC allows determining the following regularities. If the characteristic time of polymerization is greater than characteristic time of component diffusion, the dynamic range and light sensitivity of medium (recording rate) are determined by thermodynamic properties of the system. Inequalities (2) are the criterion of efficient recording in media with phase separation. They can be used to choose optimal composition components.

The microstructure that is formed during recording influences significantly the dynamic range and resolution of material. The size of dispersed phase depends on thermodynamic properties of medium, kinetic parameters of polymerization and interphase interaction forces. To control microphase structure parameters is important for the improvement of optical and holographic characteristics of photopolymers and needs further development.

Results obtained may be a basis for the development of the thermodynamic model for polymer-liquid crystal and polymer-nanoparticles structures.

\section{References}

1. W.S. Colburn, K.A. Haines, Volume hologram formation in photopolymer materials // Appl.Opt., 10(7), p. 1636-1641 (1971).

2. W.J. Tomlinson, E.A. Chandross, H.I. Weber, G.D. Aumiller, Multi-component photopolymer systems for volume phase holograms and grating devices // Appl.Opt., 15(2), p. 534541 (1976).

3. R.L. Sutherland, V.P. Tondiglia, L.V. Natarajan, T.J. Bunning, W.W. Adams, Electrically switchable volume gratings in polymer-dispersed liquid crystals // Appl.Phys.Lett., 64, p. 1074 (1994). 


\section{T. Smirnova et al.: The effect of structural-kinetic features of hologram formation on ...}

4. C.F. Van Nostrum, R.J.M. Nolte, D.J. Broer, T. Fuhram, J.H. Wendorff, Photoinduced opposite diffusion of nematic and isotropic monomers during patterned polymerization // Chem. Mater., 10, p. 135 (1998).

5. R.A. Vaia, C.L. Dennis, L.V. Natarajian, V.P. Tondiglia, D.W. Tomlin, T.J. Bunning One-step micrometer-scale organization of nano- and mesoparticles using holographic photopolymerization: a generic technique // Adv. Mater. 13(20), p. 1570-1574 (2001).

6. N. Suzuki, Ya. Tomita, T. Kojima, Holographic recording in $\mathrm{TiO}_{2}$ nanoparticle-dispersed methacrylate photopolymer films // Appl. Phys. Lett., 81(22), p. 4121-4123 (2002)

7. T.N. Smirnova, E.A. Tikhonov, E.S. Gulnazarov, Optical nonlinearity and holographic recording of stable periodic structures in polymeric photorefractive media // Proc. SPIE, 1017 p. 190-192 (1988).

8. E.S. Gulnazarov, T.N. Smirnova, E.A. Tikhonov, Recording mechanism and post-polymerizing self-amplification of holograms // Proc. SPIE, 1238, p. 235-239 (1989).

9. T.N. Smirnova, O.V. Sakhno, E.A. Tikhonov, P.V. Ezhov, V.V. Shibanov, New self-processing polymers for holographic recording over the 500-700 $\mathrm{nm}$ range. // J. Appl. Spectroscopy, 67(1), p. 29-33 (2000) (in Russian).

10. T.N. Smirnova, Structural kinetic features of formation of holograms of photopolymerizing compositions // Optics and Spectroscopy, 85(5), p. 848-852 (1998).

11. T.N. Smirnova, Thermodynamic and structural aspects of holographic recording in photopolymers // Proc. SPIE, $\mathbf{3 4 8 6}$ p. 93-102 (1998).
12. O.V Sakhno, T.N. Smirnova, Effect of thermodynamic properties of photopolymerizable compositions on their holographic characteristics // Optics and Spectroscopy, 85(6), p. 1033-1038 (1998).

13. G.M Sigalov, B.A. Rosenbergh, Equilibrium criterion of phase separation process in reactive systems // High-molecular compounds, (A)37(10), p. 1704-1708 (1995).

14. T.N. Smirnova, O.V. Sakhno, Kinetic peculiarities of holographic recording in photopolymers // Proc. SPIE, 3488, p. 267-274 (1998).

15. V.D. Solodovnik, Microcapsulation, Chemisry, Moscow (1980).

16. A.A. Askadskiy, Yu.I. Matveev, M.S. Matevosian, About polymer solubility // High-molecular compounds, (A)32(10), p. 2157-2166 (1990) (in Russian).

17. E.S. Gulnazarov, T.N. Smirnova, E.A. Tikhonov, D.V. Surovtsev, Light scattering in holograms recorded in photopolymerizing compositions //J. Appl. Spectroscopy, 51, p. 111117 (1989).

18. T.N. Smirnova, T.S. Sarbaev, E.A. Tikhonov, Real-time holographic recording of reflection gratings in photopolymer materials with varying mean refractive index and density // Optics and Spectroscopy, 83(5) p. 837-842 (1997).

19. V.P. Roschupkin, B.V. Ozerkovskiy, Yu.B. Kalmikov, G.V. Korolev, Structural physical transformations in radical polymerization processes // High-molecular compounds, (A)19(4) p. 699-706 (1977). 